\title{
Right posterior hepatic notch sign
}

\author{
Venkatraman Indiran๑, ${ }^{1}$ Kokilavani Jagannathan ${ }^{2}$ \\ ${ }^{1}$ Department of Radiodiagnosis, Sree Balaji Medical College and Hospital, 7 Works Road, Chromepet, Chennai, Tamil Nadu \\ 600044, India \\ ${ }^{2}$ VK Clinic, Chromepet, Chennai, Tamil Nadu 600044, India
}

Sharp indentation in the posteroinferior surface of right lobe of liver (between the right lobe and caudate lobe approximately at the level of right kidney) seen on axial computed tomography (CT) and magnetic resonance (MR) images [1] is known as the "right posterior hepatic notch' sign (Fig. 1). It is believed to result from atrophy of the posterior segments of right lobe of liver and hypertrophy of the caudate lobe of liver, due to hepatic fibrosis, and alteration in portal blood flow $[2,3]$.

Caudate lobe hypertrophy and a right posterior hepatic notch are more common in alcoholic cirrhosis than in viral-related cirrhosis [4]. While the right posterior hepatic notch sign has a relatively low sensitivity of $67-72 \%$ for diagnosis of cirrhosis, positive predictive value $(75-82 \%)$ and specificity $(87-98 \%)$ are higher $[1,3]$.

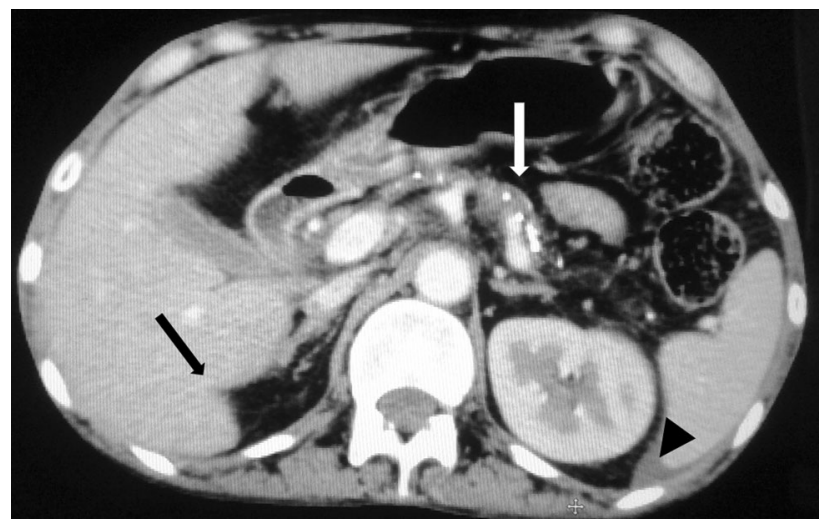

Fig. 1. Axial contrast-enhanced CT just above the level of upper pole of right kidney shows the 'right posterior hepatic notch' sign (black arrow). White arrow shows the atrophic pancreas with calcific foci. Arrowhead denotes minimal perisplenic free fluid.
Compliance with ethical standards

Funding No funding was received for this study.

Conflict of interest The authors declare that they have no conflict of interest.

Ethical approval This article does not contain any studies with animals performed by any of the author(s). All procedures performed in studies involving human participants were in accordance with the ethical standards of the institutional and/or national research committee and with the 1964 Helsinki Declaration and its later amendments or comparable ethical standards.

Informed consent Informed consent was obtained from all individual participants included in the study.

\section{References}

1. Ito K, Mitchell D (2003) Right posterior hepatic notch sign: a simple diagnostic MR finding of cirrhosis. J Magn Reson Imaging 18:561-566

2. Tan KC (2008) The right posterior hepatic notch sign. Radiology 248(1):317-318

3. Bolog N, Oancea I, Andreisek G, Mangrau A, Caruntu F (2009) Diagnosis performance of MR imaging signs of cirrhosis: the caudate to right lobe ratio, the posterior right hepatic notch, and the expanded gallbladder fossa. Curr Health Sci J. 35(1):16-22

4. Okazaki H, Ito K, Fujita T, et al. (2000) Discrimination of alcoholic from virus-induced cirrhosis on MR imaging. AJR Am J Roentgenol. 175(6):1677-1681 